\title{
Convergence to steady states for radially symmetric solutions to a quasilinear degenerate diffusive Hamilton-Jacobi equation*
}

\author{
Guy Barles ${ }^{\dagger}$ Philippe Laurençot ${ }^{\ddagger} \&$ Christian Stinner $^{\S}$
}

June 1, 2018

\begin{abstract}
Convergence to a single steady state is shown for non-negative and radially symmetric solutions to a diffusive Hamilton-Jacobi equation with homogeneous Dirichlet boundary conditions, the diffusion being the $p$-Laplacian operator, $p \geq 2$, and the source term a power of the norm of the gradient of $u$. As a first step, the radially symmetric and non-increasing stationary solutions are characterized.
\end{abstract}

Key words: convergence to steady state, degenerate parabolic equation, viscosity solutions, gradient source term

AMS Classification: 35K65; 35B40; 35J70; 49L25; 35B05

\section{Introduction}

We investigate the large time behaviour of non-negative and radially symmetric solutions to the initial-boundary value problem

$$
\left\{\begin{array}{llll}
\partial_{t} u & =\Delta_{p} u+|\nabla u|^{q}, & & x \in B, \quad t \in(0, \infty), \\
u & =0, & & x \in \partial B, \quad t \in(0, \infty), \\
u(x, 0) & =u_{0}(x), & & x \in B,
\end{array}\right.
$$

where $B:=\left\{x \in \mathbb{R}^{N}:|x|<1\right\}$ is the unit ball in $\mathbb{R}^{N}, N \geq 2$, and the $p$-Laplacian operator is defined by

$$
\Delta_{p} u=\operatorname{div}\left(|\nabla u|^{p-2} \nabla u\right) .
$$

*Partially supported by the ANR projects "Hamilton-Jacobi et théorie KAM faible" (ANR-07-BLAN-3-187245) and EVOL (ANR-08-0242)

†Laboratoire de Mathématiques et Physique Théorique, CNRS UMR 6083, Fédération Denis Poisson, Université François Rabelais, Parc de Grandmont, F-37200 Tours, France. E-mail: Guy.Barles@lmpt.univ-tours.fr

‡Institut de Mathématiques de Toulouse, CNRS UMR 5219, Université de Toulouse, F-31062 Toulouse cedex 9, France. E-mail: Philippe.Laurencot@math.univ-toulouse.fr

§Fachbereich Mathematik, Universität Duisburg-Essen, D-45117 Essen, Germany. E-mail: Christian.Stinner@uni-due.de 
We further assume the initial condition

$$
u_{0} \in W_{0}^{1, \infty}(B) \text { is radially symmetric and non-negative and } u_{0} \not \equiv 0,
$$

while the parameters $p$ and $q$ satisfy

$$
p \geq 2 \quad \text { and } \quad 0<q<p-1 .
$$

The partial differential equation in (1.1) is a second-order parabolic equation featuring a diffusion term (possibly quasilinear and degenerate if $p>2$ ) and a source term $|\nabla u|^{q}$ counteracting the effect of diffusion and depending solely on the gradient of the solution. The competition between the diffusion and the source term is already revealed by the structure of steady states to (1.1). Indeed, while it follows from [4, Theorem 1] that zero is the only steady state in $\mathcal{C}(\bar{B})$ when $p \geq 2$ and $q \geq p-1$, several steady states may exist when $p \geq 2$ and $q \in(0, p-1)$ [6, 14, 19. Another typical feature of the competition between diffusion and source is the possibility of finite time blow-up in a suitable norm, and this phenomenon has been shown to occur for (1.1) when $p=2$ and $q>2$ [17. More precisely, it is established in [17] that, when $p=2$ and $q>2$, there are classical solutions to (1.1) for which the $L^{\infty}$-norm of the gradient blows up in finite time, the $L^{\infty}$-norm of the solution remaining bounded. These solutions may actually be extended to all positive times in a unique way within the framework of viscosity solutions [5, 20, the boundary condition being also satisfied in the viscosity sense. According to the latter, the homogeneous Dirichlet boundary condition might not always be fulfilled for all times, a property which is likely to be connected with the finite time blow-up of the gradient.

Coming back to the case where $p$ and $q$ fulfil (1.3) and several steady states may exist, a complete classification of steady states seems to be out of reach when $B$ is replaced by an arbitrary open set of $\mathbb{R}^{N}$. Nevertheless, there are at least two situations in which the set of stationary solutions can be described, namely, when $N=1$ and $B=(-1,1)[14$, 19] and when $N \geq 2$ under the additional requirement that the steady states are radially symmetric and non-increasing, the latter being the first result of this paper. More precisely, we show that (1.1) has a one-parameter family of stationary solutions and that each stationary solution is characterized by the value of its maximum.

Theorem 1.1 Assume (1.3). Let $w \in W^{1, \infty}(B)$ be a radially symmetric and non-increasing viscosity solution to $-\Delta_{p} w-|\nabla w|^{q}=0$ in $B$ satisfying $w=0$ on $\partial B$. Then there is $\vartheta \in[0,1]$ such that $w=w_{\vartheta}$, where

$$
w_{\vartheta}(x):=c_{0} \int_{\max \{|x|, \vartheta\}}^{1}\left(\rho-\vartheta^{\beta} \rho^{-(\beta-1)}\right)^{1 /(p-1-q)} \mathrm{d} \rho, \quad x \in \bar{B},
$$

for $\vartheta \in[0,1]$ with

$$
\beta:=1+\frac{(N-1)(p-1-q)}{p-1}>1 \quad \text { and } \quad c_{0}:=\left(\frac{p-1-q}{(p-q) \beta}\right)^{1 /(p-1-q)}>0 .
$$

In particular, we have $w_{0}(x)=\left(c_{0} / \alpha\right)\left(1-|x|^{\alpha}\right)$ for $x \in \bar{B}$, where $\alpha:=(p-q) /(p-1-q)>1$. 
Remark 1.2 As already mentioned, for any $M \in\left[0, c_{0} / \alpha\right]$ there is one and only one $\vartheta \in[0,1]$ such that $\left\|w_{\vartheta}\right\|_{L^{\infty}(B)}=M$ as $\left\|w_{\vartheta}\right\|_{L^{\infty}(B)}$ is a decreasing function of $\vartheta \in[0,1]$. This property plays an important role in the forthcoming analysis of the large time behaviour of solutions to (1.1).

Having a precise description of the set of steady states of (1.1) at our disposal, it is natural to investigate whether they attract the dynamics of (1.1) for large times. In other words, given a solution to (1.1), does it converge to a steady state as $t \rightarrow \infty$ ? A positive answer to this question is given in [14, 19] when $N=1, B=(-1,1)$, and $p$ and $q$ fulfil (1.3). The one dimensional framework is fully exploited there as it allows the construction of a Liapunov functional by the technique developed in [21. Such a nice tool does not seem to be available here and we instead use the theory of viscosity solutions [10 and more precisely the relaxed half-limits method introduced in [7. This approach has already been used in [8, 15, 16, to investigate the large time behaviour of solutions to Hamilton-Jacobi equations and can be roughly summarized as follows: given a nonnegative and radially symmetric solution $u$ to (1.1) which is bounded in $W^{1, \infty}(B)$, the half-relaxed limits

$$
u_{*}(x):=\liminf _{(s, \varepsilon) \rightarrow(t, 0)} u\left(x, \varepsilon^{-1} s\right) \quad \text { and } \quad u^{*}(x):=\limsup _{(s, \varepsilon) \rightarrow(t, 0)} u\left(x, \varepsilon^{-1} s\right), \quad x \in \bar{B},
$$

are well-defined, do not depend on $t>0$, and are Lipschitz continuous viscosity supersolution and subsolution to

$$
-\Delta_{p} z-|\nabla z|^{q}=0 \quad \text { in } \quad B, \quad z=0 \quad \text { on } \partial B
$$

respectively, by [10, Lemma 6.1]. Clearly, $u_{*} \leq u^{*}$ on $\bar{B}$ but we cannot apply the comparison principle at this stage to conclude that $u_{*} \geq u^{*}$ on $\bar{B}$. However, additional information are available in this particular case, namely that $u_{*}$ and $u^{*}$ are both non-negative, radially symmetric, non-increasing, and have the same maximal value. Extensive use of these properties allows us to prove that $u_{*} \geq u^{*}$, from which we readily conclude that $u_{*}=u^{*}$ is a Lipschitz continuous radially symmetric and non-increasing stationary solution to (1.1). Consequently, $u_{*}=u^{*}=w_{\vartheta}$ for some $\vartheta \in[0,1]$ by Theorem 1.1 and the assumption $u_{0} \not \equiv 0$ prevents $\vartheta=1$. The convergence result we obtain actually reads as follows.

Theorem 1.3 Assume (1.2) and (1.3) and let u denote the (radially symmetric) viscosity solution to (1.1). Then there is a unique $\vartheta \in[0,1)$ such that

$$
\lim _{t \rightarrow \infty}\left\|u(t)-w_{\vartheta}\right\|_{\mathcal{C}(\bar{B})}=0 .
$$

Notice that Theorem 1.3 applies in particular in the semilinear case $p=2$ with $q \in(0,1)$ according to (1.3). Still in the semilinear case $p=2$, several results on the large time behaviour of solutions to (1.1) are also available when $q \geq 1$ and $B$ is replaced by an arbitrary open set $\Omega$ of $\mathbb{R}^{N}$, 1, 9, 18, 20, including the convergence to zero of global solutions which are bounded in $W^{1, \infty}(\Omega)$.

The analysis in this paper being restricted to radially symmetric solutions, we define $r:=|x|$ and switch between the notation $u=u(x, t)$ and $u=u(r, t)$, whenever this is convenient.

For further use, we introduce the following notations:

$$
F(s, X):=-|s|^{p-2} \operatorname{trace}(X)-(p-2)|s|^{p-4}\langle X s, s\rangle-|s|^{q} \quad \text { for }(s, X) \in \mathbb{R}^{N} \times \mathbb{R}^{N \times N},
$$


its radially symmetric counterpart

$$
f(r, \mu, \zeta):=-(p-1)|\mu|^{p-2} \zeta-\frac{N-1}{r}|\mu|^{p-2} \mu-|\mu|^{q} \quad \text { for }(r, \mu, \zeta) \in(0,1) \times \mathbb{R} \times \mathbb{R},
$$

and the radially symmetric $p$-Laplacian operator

$$
f_{0}(r, \mu, \zeta):=-(p-1)|\mu|^{p-2} \zeta-\frac{N-1}{r}|\mu|^{p-2} \mu \quad \text { for }(r, \mu, \zeta) \in(0,1) \times \mathbb{R} \times \mathbb{R} .
$$

\section{Radially symmetric and non-increasing stationary solu- tions}

In this section, we prove Theorem 1.1. that is, if $w$ is a radially symmetric, non-increasing, and Lipschitz continuous viscosity solution to the stationary equation

$$
\left\{\begin{aligned}
-\Delta_{p} w-|\nabla w|^{q}=0 & \text { in } B \\
w=0 & \text { on } \partial B
\end{aligned}\right.
$$

then $w=w_{\vartheta}$ for some $\vartheta \in[0,1]$. To this end, we first observe that, as a function of $r=|x|, w$ is a viscosity solution to $f\left(r, \partial_{r} w, \partial_{r}^{2} w\right)=0$ in $(0,1)$ with $w(1)=0$ (recall that $f$ is defined in (1.7)).

Next, as a preliminary step, let us first give a formal proof, assuming $w$ to be in $\mathcal{C}^{1}(\bar{B})$ and solving (2.1) pointwise. In particular, we will derive an identity (see (2.3) below) which turns out to be valid for viscosity solutions as we shall see later on.

As $w$ is radially symmetric and in $\mathcal{C}^{1}(\bar{B})$, we have $\partial_{r} w(0)=0$. In addition, by (2.1),

$$
\varphi(r):=r^{N-1}\left(\left|\partial_{r} w\right|^{p-2} \partial_{r} w\right)(r), \quad r \in[0,1],
$$

fulfils $\varphi \in W^{1, \infty}((0,1))$ with $\partial_{r} \varphi(r)=-r^{N-1}\left|\partial_{r} w(r)\right|^{q} \leq 0$ a.e. in $(0,1)$. Thus, $\varphi$ is a nonincreasing function in $[0,1]$. As moreover $w$ is non-increasing with $w(1)=0$, we have $\partial_{r} w(1) \leq 0$. Now, either $\partial_{r} w(1)=0$ and thus $\varphi(1)=0$. Since $\varphi$ is non-increasing with $\varphi(0)=0$, we conclude that $\varphi \equiv 0$. This implies $w=w_{1} \equiv 0$.

Or $\partial_{r} w(1)<0$, and the continuity and monotonicity of $\varphi$ warrant that there is a unique $\vartheta \in[0,1)$ such that $\varphi=0$ in $[0, \vartheta]$ and $\varphi<0$ in $(\vartheta, 1]$. Hence,

$$
\partial_{r} \varphi(r)=-r^{[(N-1)(p-1-q)] /(p-1)}|\varphi(r)|^{q /(p-1)}=-r^{\beta-1}(-\varphi(r))^{q /(p-1)} \quad \text { in } \quad(\vartheta, 1) .
$$

After integration we obtain

$$
-\frac{p-1}{p-1-q}(-\varphi(r))^{(p-1-q) /(p-1)}+\frac{1}{\beta} r^{\beta}=\gamma \quad \text { for } r \in(\vartheta, 1)
$$

with some constant $\gamma \in \mathbb{R}$. Introducing

$$
\chi(z):=\frac{p-1}{p-1-q}|z|^{p-2-q} z \quad \text { for } z \in \mathbb{R},
$$

we end up with

$$
r^{\beta-1} \chi\left(\partial_{r} w(r)\right)+\frac{1}{\beta} r^{\beta}=\gamma \quad \text { for } r \in(\vartheta, 1)
$$


as $\partial_{r} w<0$ in $(\vartheta, 1)$. Letting $r \searrow \vartheta$ implies $\gamma=\vartheta^{\beta} / \beta$ owing to $\partial_{r} w(\vartheta)=0$ and $0<q<p-1$.

Furthermore, due to $\partial_{r} w<0$ in $(\vartheta, 1)$, we have

$$
-\frac{p-1}{p-1-q}\left(r^{(N-1) /(p-1)}\left(-\partial_{r} w(r)\right)\right)^{p-1-q}=\frac{1}{\beta}\left(\vartheta^{\beta}-r^{\beta}\right) \quad \text { for } r \in(\vartheta, 1) .
$$

Hence, we conclude

$$
\partial_{r} w(r)=-\left(\frac{p-1-q}{(p-1) \beta}\left(r-\vartheta^{\beta} r^{-(\beta-1)}\right)\right)^{1 /(p-1-q)} \quad \text { for } r \in(\vartheta, 1) .
$$

Using $w(1)=0$ and the definition of $c_{0}$, a further integration implies

$$
w(r)=c_{0} \int_{r}^{1}\left(\rho-\vartheta^{\beta} \rho^{-(\beta-1)}\right)^{1 /(p-1-q)} \mathrm{d} \rho=w_{\vartheta}(r) \quad \text { for } r \in[\vartheta, 1] .
$$

Furthermore, we get $w(r)=w(\vartheta)$ for any $r \in[0, \vartheta]$ since $\partial_{r} w \equiv 0$ in $[0, \vartheta]$ and we conclude that $w=w_{\vartheta}$.

We now turn to the proof of Theorem 1.1 and first establish some preliminary results. We recall that, by the Rademacher theorem, a Lipschitz continuous function $v \in W^{1, \infty}((0,1))$ is differentiable a.e. and the measure of the differentiability set

$$
D(v):=\left\{r_{0} \in(0,1): \partial_{r} v\left(r_{0}\right) \text { exists }\right\}
$$

is thus equal to one.

Lemma 2.1 Let $v \in W^{1, \infty}((0,1))$ be a non-negative and non-increasing viscosity supersolution to

$$
f_{0}\left(r, \partial_{r} z, \partial_{r}^{2} z\right)=0 \quad \text { in }(0,1)
$$

the Hamiltonian $f_{0}$ being defined in (1.8). Then, if $r_{1} \in D(v)$ and $r_{2} \in D(v)$ are such that $r_{1}<r_{2}$, we have

$$
r_{2}^{(N-1) /(p-1)} \partial_{r} v\left(r_{2}\right) \leq r_{1}^{(N-1) /(p-1)} \partial_{r} v\left(r_{1}\right) .
$$

Proof. Take $0<r_{1}<r_{2}<1$ with $r_{1}, r_{2} \in D(v)$ and assume for contradiction that

$$
\xi_{1}:=r_{1}^{(N-1) /(p-1)} \partial_{r} v\left(r_{1}\right)<r_{2}^{(N-1) /(p-1)} \partial_{r} v\left(r_{2}\right)=: \xi_{2} .
$$

As $v$ is non-increasing we have $\xi_{2} \leq 0$. Now take $\xi_{1}<\eta_{1}<\eta_{2}<\xi_{2} \leq 0$ and define $\Phi$ by

$$
r^{(N-1) /(p-1)} \partial_{r} \Phi(r)=\eta_{1}+\left(\eta_{2}-\eta_{1}\right) \frac{r-r_{1}}{r_{2}-r_{1}}, \quad r \in\left[r_{1}, r_{2}\right]
$$

along with $\Phi\left(r_{1}\right)=0$.

On the one hand, $v-\Phi$ is continuous in $\left[r_{1}, r_{2}\right]$ and thus attains its minimum at a point $r_{0} \in\left[r_{1}, r_{2}\right]$.

On the other hand, we have

$$
\partial_{r}(v-\Phi)\left(r_{1}\right)=\frac{\xi_{1}-\eta_{1}}{r_{1}^{(N-1) /(p-1)}}<0 \quad \text { and } \quad \partial_{r}(v-\Phi)\left(r_{2}\right)=\frac{\xi_{2}-\eta_{2}}{r_{2}^{(N-1) /(p-1)}}>0
$$


so that we cannot have $r_{0}=r_{1}$ or $r_{0}=r_{2}$. Thus, $r_{0} \in\left(r_{1}, r_{2}\right)$ and, since $v$ is a viscosity supersolution to (2.4), we have

$$
-\frac{1}{r_{0}^{N-1}} \partial_{r}\left(r^{N-1}\left|\partial_{r} \Phi\right|^{p-2} \partial_{r} \Phi\right)\left(r_{0}\right) \geq 0 .
$$

Since $r^{(N-1) /(p-1)} \partial_{r} \Phi(r) \leq \eta_{2}<0$ for $r \in\left[r_{1}, r_{2}\right]$ we obtain

$$
\begin{aligned}
-\left(r^{N-1}\left|\partial_{r} \Phi\right|^{p-2} \partial_{r} \Phi\right)(r) & =r^{N-1}\left|\partial_{r} \Phi(r)\right|^{p-1}=\left(-r^{(N-1) /(p-1)} \partial_{r} \Phi(r)\right)^{p-1} \\
& =\left|\eta_{1}+\left(\eta_{2}-\eta_{1}\right) \frac{r-r_{1}}{r_{2}-r_{1}}\right|^{p-1} .
\end{aligned}
$$

Differentiating and taking $r=r_{0}$, we end up with

$$
\begin{aligned}
0 & \leq-\partial_{r}\left(r^{N-1}\left|\partial_{r} \Phi\right|^{p-2} \partial_{r} \Phi\right)\left(r_{0}\right) \\
& =(p-1)\left|\eta_{1}+\left(\eta_{2}-\eta_{1}\right) \frac{r_{0}-r_{1}}{r_{2}-r_{1}}\right|^{p-3}\left(\eta_{1}+\left(\eta_{2}-\eta_{1}\right) \frac{r_{0}-r_{1}}{r_{2}-r_{1}}\right) \frac{\eta_{2}-\eta_{1}}{r_{2}-r_{1}}<0
\end{aligned}
$$

and a contradiction.

In order to show that a viscosity solution to (2.1) satisfies (2.3), we next prove that the left-hand side of (2.3) is non-increasing for a supersolution to (2.1).

Lemma 2.2 Let $w \in W^{1, \infty}((0,1))$ be a non-increasing viscosity supersolution to $f\left(r, \partial_{r} z, \partial_{r}^{2} z\right)=0$ in $(0,1)$ such that $\|w\|_{L^{\infty}((0,1))}>0$ and $w(1)=0$, and define $r_{0} \in[0,1]$ by

$$
r_{0}:=\inf \left\{r \in(0,1]: w(r)<\|w\|_{L^{\infty}((0,1))}\right\} .
$$

If $r_{1} \in D(w)$ and $r_{2} \in D(w)$ are such that $r_{0}<r_{1}<r_{2}$, then

$$
r_{1}^{\beta-1} \chi\left(\partial_{r} w\left(r_{1}\right)\right)+\frac{r_{1}^{\beta}}{\beta} \geq r_{2}^{\beta-1} \chi\left(\partial_{r} w\left(r_{2}\right)\right)+\frac{r_{2}^{\beta}}{\beta},
$$

the parameter $\beta$ and the function $\chi$ being defined in (1.5) and (2.2), respectively.

Proof. The properties of $w$ imply $r_{0} \in[0,1)$. As $w$ is non-increasing and Lipschitz continuous, the definition of $r_{0}$ yields that there is a sequence $\left(\varrho_{n}\right)_{n \geq 1}$ such that $\varrho_{n} \in D(w), \partial_{r} w\left(\varrho_{n}\right)<0$ and $\varrho_{n} \searrow r_{0}$ as $n \rightarrow \infty$. Pick $r_{1} \in D(w) \cap\left(r_{0}, 1\right)$. For $n$ large enough, we have $r_{1}>\varrho_{n}$. Since $w$ is clearly also a supersolution to (2.4), we infer from Lemma 2.1 that

$$
r_{1}^{(N-1) /(p-1)} \partial_{r} w\left(r_{1}\right) \leq \varrho_{n}^{(N-1) /(p-1)} \partial_{r} w\left(\varrho_{n}\right)<0
$$

for $n$ large enough. Consequently,

$$
r_{1}^{(N-1) /(p-1)} \partial_{r} w\left(r_{1}\right)<0 \quad \text { for } r_{1} \in D(w) \cap\left(r_{0}, 1\right) .
$$


Assume now for contradiction that there are $r_{1}, r_{2} \in\left(r_{0}, 1\right) \cap D(w)$ such that $r_{1}<r_{2}$ and

$$
r_{1}^{\beta-1} \chi\left(\partial_{r} w\left(r_{1}\right)\right)+\frac{r_{1}^{\beta}}{\beta}<r_{2}^{\beta-1} \chi\left(\partial_{r} w\left(r_{2}\right)\right)+\frac{r_{2}^{\beta}}{\beta} .
$$

As $\partial_{r} w\left(r_{1}\right)<0$ by (2.5), we have $\chi\left(\partial_{r} w\left(r_{1}\right)\right)<0$ and we can choose two real numbers $\eta_{1}$ and $\eta_{2}$ such that

$$
r_{1}^{\beta-1} \chi\left(\partial_{r} w\left(r_{1}\right)\right)+\frac{r_{1}^{\beta}}{\beta}<\eta_{1}<\eta_{2}<r_{2}^{\beta-1} \chi\left(\partial_{r} w\left(r_{2}\right)\right)+\frac{r_{2}^{\beta}}{\beta}, \quad \eta_{1}<\frac{r_{1}^{\beta}}{\beta}
$$

and

$$
a:=1-\frac{\beta\left(\eta_{2}-\eta_{1}\right)}{r_{2}^{\beta}-r_{1}^{\beta}} \in(0,1)
$$

Indeed we first choose $\eta_{1} \in\left(r_{1}^{\beta-1} \chi\left(\partial_{r} w\left(r_{1}\right)\right)+\left(r_{1}^{\beta} / \beta\right), r_{1}^{\beta} / \beta\right)$ and then $\eta_{2}>\eta_{1}$ close enough to $\eta_{1}$ in order to have $a \in(0,1)$. Setting now

$$
A:=\eta_{1}-(1-a) \frac{r_{1}^{\beta}}{\beta}=\eta_{2}-(1-a) \frac{r_{2}^{\beta}}{\beta},
$$

let $\Phi$ denote the solution to

$$
r^{\beta-1} \chi\left(\partial_{r} \Phi(r)\right)+a \frac{r^{\beta}}{\beta}=A, \quad r \in\left[r_{1}, r_{2}\right],
$$

such that $\Phi\left(r_{1}\right)=0$. Observe that the choice of $a$ and $A$ ensure that and

$$
r_{i}^{\beta-1} \chi\left(\partial_{r} \Phi\left(r_{i}\right)\right)+\frac{r_{i}^{\beta}}{\beta}=\eta_{i} \quad \text { for } i=1,2 .
$$

Due to

$$
A-a \frac{r_{1}^{\beta}}{\beta}=\eta_{1}-\frac{r_{1}^{\beta}}{\beta}<0
$$

we conclude by (2.6) that

$$
\chi\left(\partial_{r} \Phi(r)\right)=r^{-(\beta-1)}\left(A-a \frac{r^{\beta}}{\beta}\right) \leq r^{-(\beta-1)}\left(A-a \frac{r_{1}^{\beta}}{\beta}\right)<0 \quad \text { for } r \in\left[r_{1}, r_{2}\right] .
$$

This implies that $\partial_{r} \Phi(r)<0$ for $r \in\left[r_{1}, r_{2}\right]$, so that $\Phi \in \mathcal{C}^{2}\left(\left[r_{1}, r_{2}\right]\right)$ by (2.6). In addition,

$$
\left(-\partial_{r} \Phi(r)\right)^{p-1-q}=\frac{p-1-q}{p-1}\left(\frac{a}{\beta} r-A r^{-(\beta-1)}\right), \quad r \in\left[r_{1}, r_{2}\right],
$$

hence

$$
\partial_{r} \Phi(r)=-\left[\frac{p-1-q}{p-1}\left(\frac{a}{\beta} r-A r^{-(\beta-1)}\right)\right]^{1 /(p-1-q)}, \quad r \in\left[r_{1}, r_{2}\right]
$$


Furthermore, due to (2.7) and the choice of $\eta_{1}$, we obtain

$$
r_{1}^{\beta-1} \chi\left(\partial_{r} w\left(r_{1}\right)\right)+\frac{r_{1}^{\beta}}{\beta}<\eta_{1}=r_{1}^{\beta-1} \chi\left(\partial_{r} \Phi\left(r_{1}\right)\right)+\frac{r_{1}^{\beta}}{\beta} .
$$

This implies $\chi\left(\partial_{r} w\left(r_{1}\right)\right)<\chi\left(\partial_{r} \Phi\left(r_{1}\right)\right)$ and, since $\chi$ is increasing,

$$
\partial_{r} w\left(r_{1}\right)<\partial_{r} \Phi\left(r_{1}\right) \text {. }
$$

Similarly, we conclude

$$
\partial_{r} w\left(r_{2}\right)>\partial_{r} \Phi\left(r_{2}\right) .
$$

Now $w-\Phi$ is a continuous function in $\left[r_{1}, r_{2}\right]$ and thus attains its minimum at some $r_{m} \in\left[r_{1}, r_{2}\right]$. The above two inequalities prevent $r_{m}$ to be equal to $r_{1}$ or $r_{2}$ and, since $w$ is a viscosity supersolution to $f\left(r, \partial_{r} v, \partial_{r}^{2} v\right)=0$ in $(0,1)$, we have

$$
-\frac{1}{r_{m}^{N-1}} \partial_{r}\left(r^{N-1}\left|\partial_{r} \Phi(r)\right|^{p-2} \partial_{r} \Phi(r)\right)\left(r_{m}\right)-\left|\partial_{r} \Phi\left(r_{m}\right)\right|^{q} \geq 0 .
$$

But as $\partial_{r} \Phi<0$, (2.6) implies

$$
\begin{aligned}
& -\partial_{r}\left(r^{N-1}\left|\partial_{r} \Phi(r)\right|^{p-2} \partial_{r} \Phi(r)\right)=\partial_{r}\left(r^{N-1}\left|\partial_{r} \Phi(r)\right|^{p-1}\right) \\
= & \partial_{r}\left(\left|\frac{p-1-q}{p-1} r^{\beta-1} \chi\left(\partial_{r} \Phi(r)\right)\right|(p-1) /(p-1-q)\right. \\
= & -a r^{\beta-1}\left|\frac{p-1-q}{p-1} r^{\beta-1} \chi\left(\partial_{r} \Phi(r)\right)\right|^{[(p-1) /(p-1-q)]-2}\left(\frac{p-1-q}{p-1} r^{\beta-1} \chi\left(\partial_{r} \Phi(r)\right)\right) \\
= & a r^{(\beta-1)(p-1) /(p-1-q)}\left|\frac{p-1-q}{p-1} \chi\left(\partial_{r} \Phi(r)\right)\right|^{[(p-1) /(p-1-q)]-1} \\
= & a r^{N-1}\left|\partial_{r} \Phi(r)\right|^{q} \quad \text { for } r \in\left[r_{1}, r_{2}\right],
\end{aligned}
$$

so that

$$
-\frac{1}{r_{m}^{N-1}} \partial_{r}\left(r^{N-1}\left|\partial_{r} \Phi(r)\right|^{p-2} \partial_{r} \Phi(r)\right)\left(r_{m}\right)-\left|\partial_{r} \Phi\left(r_{m}\right)\right|^{q}=(a-1)\left|\partial_{r} \Phi\left(r_{m}\right)\right|^{q}<0
$$

since $a<1$, and a contradiction.

In a similar way we now establish that the left-hand side of (2.3) is non-decreasing for viscosity subsolutions to (2.1).

Lemma 2.3 Let $w \in W^{1, \infty}((0,1))$ be a non-increasing viscosity subsolution to $f\left(r, \partial_{r} z, \partial_{r}^{2} z\right)=0$ in $(0,1)$ such that $\|w\|_{L^{\infty}((0,1))}>0$ and $w(1)=0$, and define $r_{0} \in[0,1]$ by

$$
r_{0}:=\inf \left\{r \in(0,1]: w(r)<\|w\|_{L^{\infty}((0,1))}\right\} .
$$

If $r_{1} \in D(w)$ and $r_{2} \in D(w)$ are such that $r_{0}<r_{1}<r_{2}$, then

$$
r_{1}^{\beta-1} \chi\left(\partial_{r} w\left(r_{1}\right)\right)+\frac{r_{1}^{\beta}}{\beta} \leq r_{2}^{\beta-1} \chi\left(\partial_{r} w\left(r_{2}\right)\right)+\frac{r_{2}^{\beta}}{\beta} .
$$


Proof. The properties of $w$ imply $r_{0} \in[0,1)$. Assume for contradiction that there are $r_{1}, r_{2} \in$ $\left(r_{0}, 1\right) \cap D(w)$ such that $r_{1}<r_{2}$ and

$$
r_{1}^{\beta-1} \chi\left(\partial_{r} w\left(r_{1}\right)\right)+\frac{r_{1}^{\beta}}{\beta}>r_{2}^{\beta-1} \chi\left(\partial_{r} w\left(r_{2}\right)\right)+\frac{r_{2}^{\beta}}{\beta} .
$$

We may then choose $\eta_{1}, \eta_{2} \in \mathbb{R}$ such that

$$
r_{1}^{\beta-1} \chi\left(\partial_{r} w\left(r_{1}\right)\right)+\frac{r_{1}^{\beta}}{\beta}>\eta_{1}>\eta_{2}>r_{2}^{\beta-1} \chi\left(\partial_{r} w\left(r_{2}\right)\right)+\frac{r_{2}^{\beta}}{\beta},
$$

and define

$$
a:=1+\frac{\beta\left(\eta_{1}-\eta_{2}\right)}{r_{2}^{\beta}-r_{1}^{\beta}}>1 \quad \text { and } \quad A:=\eta_{1}+(a-1) \frac{r_{1}^{\beta}}{\beta}=\eta_{2}+(a-1) \frac{r_{2}^{\beta}}{\beta} .
$$

Let $\Phi$ denote the solution to

$$
r^{\beta-1} \chi\left(\partial_{r} \Phi(r)\right)+a \frac{r^{\beta}}{\beta}=A, \quad r \in\left[r_{1}, r_{2}\right],
$$

such that $\Phi\left(r_{1}\right)=0$. Thanks to the choice of $a$ and $A$, we have

$$
r_{i}^{\beta-1} \chi\left(\partial_{r} \Phi\left(r_{i}\right)\right)+\frac{r_{i}^{\beta}}{\beta}=\eta_{i} \quad \text { for } i=1,2,
$$

and the monotonicity of $w$ implies that

$$
A-a \frac{r_{1}^{\beta}}{\beta}=\eta_{1}-\frac{r_{1}^{\beta}}{\beta}<r_{1}^{\beta-1} \chi\left(\partial_{r} w\left(r_{1}\right)\right) \leq 0 .
$$

Consequently,

$$
\chi\left(\partial_{r} \Phi(r)\right)=r^{-(\beta-1)}\left(A-a \frac{r^{\beta}}{\beta}\right) \leq r^{-(\beta-1)}\left(A-a \frac{r_{1}^{\beta}}{\beta}\right)<0 \quad \text { for } r \in\left[r_{1}, r_{2}\right],
$$

hence $\partial_{r} \Phi(r)<0$ for $r \in\left[r_{1}, r_{2}\right]$. We then conclude from (2.9) that $\Phi \in \mathcal{C}^{2}\left(\left[r_{1}, r_{2}\right]\right)$. Furthermore, due to (2.10), the choices of $\eta_{1}$ and $\eta_{2}$, and the monotonicity of $\chi$, we obtain

$$
\partial_{r} w\left(r_{1}\right)>\partial_{r} \Phi\left(r_{1}\right) \quad \text { and } \quad \partial_{r} w\left(r_{2}\right)<\partial_{r} \Phi\left(r_{2}\right) .
$$

Now $w-\Phi$ is a continuous function in $\left[r_{1}, r_{2}\right]$ and thus attains its maximum at some point $r_{m} \in$ $\left[r_{1}, r_{2}\right]$. The above two inequalities prevent $r_{m}$ to be equal to $r_{1}$ or $r_{2}$ and, since $w$ is a viscosity subsolution to $f\left(r, \partial_{r} v, \partial_{r}^{2} v\right)=0$ in $(0,1)$, we have

$$
-\frac{1}{r_{m}^{N-1}} \partial_{r}\left(r^{N-1}\left|\partial_{r} \Phi(r)\right|^{p-2} \partial_{r} \Phi(r)\right)\left(r_{m}\right)-\left|\partial_{r} \Phi\left(r_{m}\right)\right|^{q} \leq 0 .
$$

But, owing to $\partial_{r} \Phi(r)<0$, (2.9) and $a>1$, we conclude similarly to (2.8) that

$$
-\frac{1}{r_{m}^{N-1}} \partial_{r}\left(r^{N-1}\left|\partial_{r} \Phi(r)\right|^{p-2} \partial_{r} \Phi(r)\right)\left(r_{m}\right)-\left|\partial_{r} \Phi\left(r_{m}\right)\right|^{q}=(a-1)\left|\partial_{r} \Phi\left(r_{m}\right)\right|^{q}>0
$$


and end up with a contradiction.

We are now in a position to prove Theorem 1.1 The keystone of the proof is that, according to Lemma 2.2 and Lemma 2.3 any non-increasing viscosity solution to $f\left(r, \partial_{r} v, \partial_{r}^{2} v\right)=0$ in $(0,1)$ satisfying $w(1)=0$ has to fulfil (2.3).

Proof of TheOREM 1.1.

Let $w \in W^{1, \infty}((0,1))$ be a non-increasing viscosity solution to $f\left(r, \partial_{r} v, \partial_{r}^{2} v\right)=0$ in $(0,1)$ satisfying $w(1)=0$. Either $w \equiv 0=w_{1}$ or $M:=\|w\|_{L^{\infty}((0,1))}>0$ and we define $r_{0} \in[0,1)$ by

$$
r_{0}:=\inf \{r \in(0,1]: w(r)<M\} .
$$

Now, owing to Lemma 2.2 and Lemma 2.3 , there is a constant $\gamma \in \mathbb{R}$ such that

$$
r^{\beta-1} \chi\left(\partial_{r} w(r)\right)+\frac{r^{\beta}}{\beta}=\gamma
$$

for any $r \in\left(r_{0}, 1\right) \cap D(w)$ and thus a.e. in $\left(r_{0}, 1\right)$. Combining the monotonicity of $w$ and $\chi$ with (2.11), we moreover deduce that

$$
\gamma \leq \frac{r_{0}^{\beta}}{\beta}
$$

and

$$
\partial_{r} w(r)=-\left[\frac{p-1-q}{p-1}\left(\frac{r}{\beta}-\gamma r^{-(\beta-1)}\right)\right]^{1 /(p-1-q)} \quad \text { for a.e. } r \in\left(r_{0}, 1\right) .
$$

Integrating and using the boundary condition $w(1)=0$, we obtain

$$
w(r)=\int_{r}^{1}\left[\frac{p-1-q}{(p-1) \beta}\left(\rho-\gamma \beta \rho^{-(\beta-1)}\right)\right]^{1 /(p-1-q)} \mathrm{d} \rho \quad \text { for any } r \in\left[r_{0}, 1\right] .
$$

Recalling $w(r) \equiv M$ for $r \in\left[0, r_{0}\right]$ and the definition of $c_{0}$, we conclude

$$
w(r)=c_{0} \int_{\max \left\{r, r_{0}\right\}}^{1}\left(\rho-\gamma \beta \rho^{-(\beta-1)}\right)^{1 /(p-1-q)} \mathrm{d} \rho, \quad r \in[0,1] .
$$

It remains to show that $\gamma=r_{0}^{\beta} / \beta$ in order to obtain that $w=w_{r_{0}}$.

Consider first the case $r_{0}=0$. Since $\beta>1$, the Lipschitz continuity of $w$ yields $\gamma=0=r_{0}^{\beta} / \beta$ by letting $r \searrow 0$ in (2.11).

Next, if $r_{0} \in(0,1)$, we assume for contradiction that $\gamma<r_{0}^{\beta} / \beta$. Then we fix $\vartheta \in\left[0, r_{0}\right)$ such that $\gamma<\vartheta^{\beta} / \beta$ and choose $\Lambda>1$ such that

$$
\Lambda^{p-1-q}<1+\vartheta^{\beta}-\gamma \beta .
$$

This choice of $\Lambda$ implies that the function

$$
g(r):=\left(1-\gamma \beta r^{-\beta}\right)-\Lambda^{p-1-q}\left(1-\vartheta^{\beta} r^{-\beta}\right), \quad r \in\left(r_{0}, 1\right)
$$


satisfies

$$
g^{\prime}(r)=\beta^{2} r^{-\beta-1}\left(\gamma-\Lambda^{p-1-q} \frac{\vartheta^{\beta}}{\beta}\right) \leq \beta^{2} r^{-\beta-1}\left(\gamma-\frac{\vartheta^{\beta}}{\beta}\right)<0, \quad r \in\left(r_{0}, 1\right)
$$

and thus

$$
g(r) \geq g(1) \geq 1-\gamma \beta-\Lambda^{p-1-q}+\vartheta^{\beta}>0, \quad r \in\left[r_{0}, 1\right] .
$$

Consequently,

$$
\left(1-\gamma \beta r^{-\beta}\right)>\Lambda^{p-1-q}\left(1-\vartheta^{\beta} r^{-\beta}\right), \quad r \in\left[r_{0}, 1\right],
$$

and it follows from (2.13) that

$$
\begin{aligned}
\partial_{r} w(r) & =-c_{0} r^{1 /(p-1-q)}\left(1-\gamma \beta r^{-\beta}\right)^{1 /(p-1-q)} \\
& <-c_{0} r^{1 /(p-1-q)} \Lambda\left(1-\vartheta^{\beta} r^{-\beta}\right)^{1 /(p-1-q)}=\Lambda \partial_{r} w_{\vartheta}(r), \quad r \in\left(r_{0}, 1\right) .
\end{aligned}
$$

In particular, $w(r)-\Lambda w_{\vartheta}(r) \leq w\left(r_{0}\right)-\Lambda w_{\vartheta}\left(r_{0}\right)$ for $r \in\left[r_{0}, 1\right]$. Furthermore,

$$
w(r)-\Lambda w_{\vartheta}(r)=w\left(r_{0}\right)-\Lambda w_{\vartheta}(r) \leq w\left(r_{0}\right)-\Lambda w_{\vartheta}\left(r_{0}\right), \quad r \in\left[0, r_{0}\right],
$$

thanks to the monotonicity of $w_{\vartheta}$, and the function $w-\Lambda w_{\vartheta}$ has a global maximum at $r_{0}$. Since $w_{\vartheta} \in \mathcal{C}^{2}((\vartheta, 1)), \vartheta<r_{0}$, and $w$ is a viscosity subsolution to $f\left(r, \partial_{r} v, \partial_{r}^{2} v\right)=0$ in $(0,1)$, we conclude that

$$
f\left(r_{0}, \partial_{r}\left(\Lambda w_{\vartheta}\right)\left(r_{0}\right), \partial_{r}^{2}\left(\Lambda w_{\vartheta}\right)\left(r_{0}\right)\right) \leq 0
$$

However, as $\Lambda>1$ and $\vartheta<r_{0}$, we clearly have

$$
f\left(r_{0}, \partial_{r}\left(\Lambda w_{\vartheta}\right)\left(r_{0}\right), \partial_{r}^{2}\left(\Lambda w_{\vartheta}\right)\left(r_{0}\right)\right)=\left(\Lambda^{p-1}-\Lambda^{q}\right)\left|\partial_{r} w_{\vartheta}\left(r_{0}\right)\right|^{q}>0,
$$

and the contradiction. Therefore, $\gamma=r_{0}^{\beta} / \beta$ and $w=w_{r_{0}}$, which completes the proof.

\section{Some properties of solutions to (1.1)}

We now focus on time-dependent solutions to (1.1) and establish some qualitative properties of non-negative and radially symmetric viscosity solutions to (1.1) which are needed to analyse their large time behaviour.

Proposition 3.1 Assume that $u_{0}, p$, and $q$ fulfil 1.2) and (1.3). There is a unique non-negative viscosity solution $u \in \mathcal{C}(\bar{B} \times[0, \infty))$ to (1.1) such that $u(x, t)=0$ for $x \in \partial B$ and $x \longmapsto u(x, t)$ is radially symmetric and belongs to $W^{1, \infty}(B)$ for all $t \geq 0$. In addition, there is a constant $A_{0}>0$ depending only on $p, q$, and $u_{0}$, and a decreasing function $W \in \mathcal{C}^{1}([0, \infty))$ such that

$$
0 \leq u(x, t) \leq A_{0} \quad \text { and } \quad-A_{0} \leq \nabla u(x, t) \cdot \frac{x}{|x|} \leq W(t), \quad(x, t) \in \bar{B} \times[0, \infty),
$$

and $W(t) \longrightarrow 0$ as $t \rightarrow \infty$. 
Proof. We first derive the expected properties on suitable approximations to (1.1) which we introduce now. For $\varepsilon \in(0,1)$, let $a_{\varepsilon} \in \mathcal{C}^{\infty}([0, \infty))$ and $b_{\varepsilon} \in \mathcal{C}^{\infty}([0, \infty))$ be two functions such that

- $a_{\varepsilon}$ is bounded and increasing and $a_{\varepsilon}(\xi):=\left(\varepsilon^{2}+\xi\right)^{(p-2) / 2}$ for $\xi \in\left[0, \varepsilon^{-1}\right]$,

- $b_{\varepsilon}$ is increasing, Lipschitz continuous, and $b_{\varepsilon}(\xi):=\left(\varepsilon^{2}+\xi\right)^{q / 2}-\varepsilon^{q}$ for $\xi \in\left[0, \varepsilon^{-1}\right]$.

In addition, owing to the properties (1.2) of $u_{0}$, there exists a sequence $\left(u_{0 \varepsilon}\right)_{\varepsilon \in(0,1)}$ of non-negative and radially symmetric functions in $\mathcal{C}^{\infty}(\bar{B})$ such that

$$
\left\|u_{0 \varepsilon}\right\|_{L^{\infty}(B)} \leq\left\|u_{0}\right\|_{L^{\infty}(B)}+\varepsilon, \quad\left\|\nabla u_{0 \varepsilon}\right\|_{L^{\infty}(B)} \leq 2\left\|\nabla u_{0}\right\|_{L^{\infty}(B)},
$$

and

$$
\lim _{\varepsilon \rightarrow 0}\left\|u_{0 \varepsilon}-u_{0}\right\|_{\mathcal{C}(\bar{B})}=0 .
$$

Fix $\varepsilon \in(0,1)$. According to the properties of $a_{\varepsilon}, b_{\varepsilon}$, and $u_{0 \varepsilon}$, it follows from [13] that the initialboundary value problem

$$
\left\{\begin{array}{llll}
\partial_{t} u_{\varepsilon} & =\operatorname{div}\left(a_{\varepsilon}\left(\left|\nabla u_{\varepsilon}\right|^{2}\right) \nabla u_{\varepsilon}\right)+b_{\varepsilon}\left(\left|\nabla u_{\varepsilon}\right|^{2}\right), & & x \in B, \\
u_{\varepsilon} & =0, & & x \in \partial B, \quad t \in(0, \infty), \\
\left.u_{\varepsilon}\right|_{t=0} & =u_{0 \varepsilon}, & & x \in B,
\end{array}\right.
$$

has a unique non-negative classical solution $u_{\varepsilon}$. In addition, $x \longmapsto u_{\varepsilon}(t, x)$ is radially symmetric for every $t \geq 0$ and the comparison principle entails that

$$
0 \leq u_{\varepsilon}(x, t) \leq\left\|u_{0 \varepsilon}\right\|_{L^{\infty}(B)} \leq\left\|u_{0}\right\|_{L^{\infty}(B)}+\varepsilon, \quad(x, t) \in \bar{B} \times[0, \infty) .
$$

We next derive some estimates on the gradient of $u_{\varepsilon}$ and begin with the normal trace $\partial_{r} u_{\varepsilon}(1, t)$. Let $\mathcal{L}_{\varepsilon}$ be the parabolic operator

$$
\mathcal{L}_{\varepsilon} z:=\partial_{t} z-\frac{1}{r^{N-1}} \partial_{r}\left(r^{N-1} a_{\varepsilon}\left(\left|\partial_{r} z\right|^{2}\right) \partial_{r} z\right)-b_{\varepsilon}\left(\left|\partial_{r} z\right|^{2}\right), \quad(r, t) \in(0,1) \times(0, \infty),
$$

and fix

$$
A_{0} \in\left(\sqrt{3} \varepsilon, \varepsilon^{-1 / 2}\right) \text { such that } A_{0} \geq 2^{1 /(p-1-q)}+2\left(1+\left\|u_{0}\right\|_{L^{\infty}(B)}+\left\|\nabla u_{0}\right\|_{L^{\infty}(B)}\right) .
$$

Then, thanks to the properties of $a_{\varepsilon}, b_{\varepsilon}$, and (3.5), the function $\psi$ defined by $\psi(r):=A_{0}(1-r)$ for $r \in[0,1]$ satisfies

$$
\begin{aligned}
\mathcal{L}_{\varepsilon} \psi(r) & =\frac{1}{r^{N-1}} \partial_{r}\left(r^{N-1} a_{\varepsilon}\left(A_{0}^{2}\right) A_{0}\right)-b_{\varepsilon}\left(A_{0}^{2}\right)=\frac{N-1}{r} a_{\varepsilon}\left(A_{0}^{2}\right) A_{0}-b_{\varepsilon}\left(A_{0}^{2}\right) \\
& \geq\left(\varepsilon^{2}+A_{0}^{2}\right)^{(p-2) / 2} A_{0}-\left(\varepsilon^{2}+A_{0}^{2}\right)^{q / 2}+\varepsilon^{q} \\
& \geq\left(\varepsilon^{2}+A_{0}^{2}\right)^{(p-2) / 2}\left(\sqrt{\varepsilon^{2}+A_{0}^{2}}-\varepsilon\right)-\left(\varepsilon^{2}+A_{0}^{2}\right)^{q / 2} \\
& \geq\left(\varepsilon^{2}+A_{0}^{2}\right)^{(p-1) / 2}\left(1-\frac{\varepsilon}{\sqrt{\varepsilon^{2}+A_{0}^{2}}}\right)-\left(\varepsilon^{2}+A_{0}^{2}\right)^{q / 2} \\
& \geq \frac{1}{2}\left(\varepsilon^{2}+A_{0}^{2}\right)^{(p-1) / 2}-\left(\varepsilon^{2}+A_{0}^{2}\right)^{q / 2} \geq 0, \quad r \in(0,1] .
\end{aligned}
$$


Furthermore, (3.2), (3.4), and (3.5) entail that

$$
u_{\varepsilon}\left(\frac{1}{2}, t\right) \leq 1+\left\|u_{0}\right\|_{L^{\infty}(B)} \leq \frac{A_{0}}{2}=\psi\left(\frac{1}{2}\right), \quad t \geq 0,
$$

and

$$
u_{0 \varepsilon}(r)=-\int_{r}^{1} \partial_{r} u_{0 \varepsilon}(\varrho) d \varrho \leq 2\left\|\nabla u_{0}\right\|_{\infty}(1-r) \leq \psi(r), \quad r \in\left(\frac{1}{2}, 1\right) .
$$

Since $\mathcal{L}_{\varepsilon} u_{\varepsilon}=0$ in $(1 / 2,1) \times(0, \infty)$, the comparison principle ensures that $u_{\varepsilon}(r, t) \leq A_{0}(1-r)$ for $(r, t) \in(1 / 2,1) \times(0, \infty)$. Since $u_{\varepsilon}(1, t)=0$, this implies in particular that $0 \leq-\partial_{r} u_{\varepsilon}(1, t) \leq A_{0}$ for $t \geq 0$. Recalling that $u_{\varepsilon}(t)$ is radially symmetric and smooth, we thus have

$$
-A_{0} \leq \partial_{r} u_{\varepsilon}(1, t) \leq 0=\partial_{r} u_{\varepsilon}(0, t), \quad t \geq 0 .
$$

We next estimate the gradient of $u_{\varepsilon}$ in $B$. For that purpose, we introduce the parabolic operator

$$
\begin{aligned}
\mathcal{M}_{\varepsilon} z:= & \partial_{t} z-\partial_{r}\left[\left(a_{\varepsilon}\left(z^{2}\right)+2 a_{\varepsilon}^{\prime}\left(z^{2}\right) z^{2}\right) \partial_{r} z\right] \\
& -\left[\frac{N-1}{r}\left(a_{\varepsilon}\left(z^{2}\right)+2 a_{\varepsilon}^{\prime}\left(z^{2}\right) z^{2}\right)+2 b_{\varepsilon}^{\prime}\left(z^{2}\right) z^{2}\right] \partial_{r} z+\frac{N-1}{r^{2}} a_{\varepsilon}\left(z^{2}\right) z
\end{aligned}
$$

for $(r, t) \in(0,1) \times(0, \infty)$ and readily deduce from (3.3) that

$$
\mathcal{M}_{\varepsilon} \partial_{r} u_{\varepsilon}=0 \quad \text { in }(0,1) \times(0, \infty) .
$$

Observe next that $\partial_{r} u_{\varepsilon}(r, 0) \geq-2\left\|\nabla u_{0}\right\|_{L^{\infty}(B)} \geq-A_{0}$ by (3.2) and (3.5) and

$$
\mathcal{M}_{\varepsilon}\left(-A_{0}\right)=-\frac{N-1}{r^{2}} a_{\varepsilon}\left(A_{0}^{2}\right) A_{0} \leq 0,
$$

which, together with (3.6), (3.7), and the comparison principle implies that

$$
-A_{0} \leq \partial_{r} u_{\varepsilon}(r, t), \quad(r, t) \in[0,1] \times[0, \infty) .
$$

Finally, let $W_{\varepsilon} \in \mathcal{C}^{1}([0, \infty))$ be the solution to the ordinary differential equation

$$
\frac{d W_{\varepsilon}}{d t}+(N-1) a_{\varepsilon}\left(W_{\varepsilon}^{2}\right) W_{\varepsilon}=0, \quad W_{\varepsilon}(0)=2\left\|\nabla u_{0}\right\|_{L^{\infty}(B)} .
$$

Then $W_{\varepsilon}$ is positive and decreasing, $W_{\varepsilon}(0) \geq \partial_{r} u_{\varepsilon}(r, 0)$ for $r \in(0,1)$ by (3.2), and $\mathcal{M}_{\varepsilon} W_{\varepsilon} \geq 0$ in $(0,1) \times(0, \infty)$ by (3.9). Recalling (3.7), we deduce from the comparison principle that

$$
\partial_{r} u_{\varepsilon}(r, t) \leq W_{\varepsilon}(t), \quad(r, t) \in[0,1] \times[0, \infty) .
$$

Finally, we argue as in [12, Lemma 5] to deduce from (3.3), (3.4), (3.8), and (3.10) that there is a constant $C$ depending on $\left\|\nabla u_{0}\right\|_{L^{\infty}(B)}, p, q$, and $N$, such that

$$
\left|u_{\varepsilon}\left(x, t_{1}\right)-u_{\varepsilon}\left(x, t_{2}\right)\right| \leq C\left(\left|t_{1}-t_{2}\right|+\left|t_{1}-t_{2}\right|^{1 / 2}\right)
$$


for any $x \in \bar{B}, t_{1}, t_{2} \in[0, \infty)$ and $\varepsilon \in(0,1)$. Indeed, consider $t_{1} \neq t_{2}$ and set $\tau:=\left|t_{1}-t_{2}\right|^{1 / 2}>0$ and $L:=\max \left\{A_{0}, 2\left\|\nabla u_{0}\right\|_{L^{\infty}(B)}\right\}$. Since (3.8), (3.10), and the Dirichlet boundary conditions imply that $\left|u_{\varepsilon}(x, t)\right| \leq L \operatorname{dist}(x, \partial B)$ for $(x, t) \in \bar{B} \times[0, \infty)$, we have

$$
\left|u_{\varepsilon}\left(x_{0}, t_{1}\right)-u_{\varepsilon}\left(x_{0}, t_{2}\right)\right| \leq 2 L \operatorname{dist}\left(x_{0}, \partial B\right) \leq 2 L \tau \quad \text { if } \quad \operatorname{dist}\left(x_{0}, \partial B\right) \leq \tau .
$$

If $\operatorname{dist}\left(x_{0}, \partial B\right)>\tau$ and $\varepsilon \in(0,1 / L)$, we infer from (3.3), the properties of $\left(a_{\varepsilon}, b_{\varepsilon}\right)$, and $\left|\nabla u_{\varepsilon}\right| \leq L$ in $B \times[0, \infty)$ that

$$
\begin{aligned}
& \left|u_{\varepsilon}\left(x_{0}, t_{1}\right)-u_{\varepsilon}\left(x_{0}, t_{2}\right)\right|=\frac{1}{|B| \tau^{N}}\left|\int_{\left\{\left|x-x_{0}\right|<\tau\right\}}\left(u_{\varepsilon}\left(x_{0}, t_{1}\right)-u_{\varepsilon}\left(x_{0}, t_{2}\right)\right) \mathrm{d} x\right| \\
& =\left.\left.\frac{1}{|B| \tau^{N}}\right|_{\left\{\left|x-x_{0}\right|<\tau\right\}}\left(u_{\varepsilon}(x, t)-u_{\varepsilon}\left(x_{0}, t\right)\right) \mathrm{d} x\right|_{t=t_{1}} ^{t=t_{2}} \\
& -\int_{t_{1}}^{t_{2}} \int_{\left\{\left|x-x_{0}\right|<\tau\right\}} \partial_{t} u_{\varepsilon}(x, t) \mathrm{d} x \mathrm{~d} t \mid \\
& \leq \frac{2 L}{|B| \tau^{N}} \int_{\left\{\left|x-x_{0}\right|<\tau\right\}}\left|x-x_{0}\right| \mathrm{d} x \\
& +\frac{1}{|B| \tau^{N}}\left|\int_{t_{1}}^{t_{2}} \int_{\left\{\left|x-x_{0}\right|<\tau\right\}}\left[\operatorname{div}\left(a_{\varepsilon}\left(\left|\nabla u_{\varepsilon}\right|^{2}\right) \nabla u_{\varepsilon}\right)+b_{\varepsilon}\left(\left|\nabla u_{\varepsilon}\right|^{2}\right)\right](x, t) \mathrm{d} x \mathrm{~d} t\right| \\
& \leq \frac{2 L N}{N+1} \tau+\frac{1}{|B| \tau^{N}}\left|\int_{t_{1}}^{t_{2}} \int_{\left\{\left|x-x_{0}\right|<\tau\right\}}\left(\varepsilon^{2}+\left|\nabla u_{\varepsilon}\right|^{2}\right)^{q / 2}(x, t) \mathrm{d} x \mathrm{~d} t\right| \\
& +\frac{1}{|B| \tau^{N}}\left|\int_{t_{1}}^{t_{2}} \int_{\left\{\left|x-x_{0}\right|=\tau\right\}}\left[a_{\varepsilon}\left(\left|\nabla u_{\varepsilon}\right|^{2}\right)\left|\nabla u_{\varepsilon}\right|\right](y, t) \mathrm{d} S \mathrm{~d} t\right| \\
& \leq \frac{2 L N}{N+1} \tau+\left(1+L^{2}\right)^{q / 2}\left|t_{1}-t_{2}\right|+\frac{N}{\tau}\left(1+L^{2}\right)^{(p-2) / 2} L\left|t_{1}-t_{2}\right| \\
& \leq \frac{2 L N}{N+1} \tau+\left(1+L^{2}\right)^{q / 2} \tau^{2}+N\left(1+L^{2}\right)^{p / 2} \tau .
\end{aligned}
$$

Combining (3.12) and the above estimate gives the claim (3.11).

We can now pass to the limit as $\varepsilon \rightarrow 0$. Owing to (3.4), (3.8), (3.10), and (3.11), $\left(u_{\varepsilon}\right)_{\varepsilon}$ is bounded in, say, $C^{0,1 / 2}(B \times(0, \infty))$ because the uniform Lipschitz continuity in $r$ implies a uniform $C^{0,1 / 2}$ bound in $r$; thus $\left(u_{\varepsilon}\right)_{\varepsilon}$ is relatively compact in $\mathcal{C}(\bar{B} \times[0, T])$ for all $T>0$. It follows from the stability theorem [10, Section 6] and the comparison principle for (1.1) [11, Theorem 2.1] that $\left(u_{\varepsilon}\right)_{\varepsilon}$ converges uniformly towards the unique viscosity solution $u$ to (1.1) on compact subsets of $\bar{B} \times[0, \infty)$. The properties of $u$ and the bounds listed in Proposition 3.1 then readily follow from 
this convergence, the properties of $u_{\varepsilon}$, (3.4), (3.8), and (3.10), the function $W$ being the solution to the ordinary differential equation

$$
\frac{d W}{d t}+(N-1)|W|^{p-2} W=0, \quad W(0)=2\left\|\nabla u_{0}\right\|_{L^{\infty}(B)} .
$$

In fact, $W(t)=\left(W(0)^{2-p}+(p-2)(N-1) t\right)^{-1 /(p-2)}$ for $t \geq 0$ and $W$ is obviously positive, decreasing and converges to zero as $t \rightarrow \infty$.

By (3.1), the trajectory $\{u(t): t \geq 0\}$ of the solution $u$ to (1.1) is bounded in $L^{\infty}(B)$. More precise information are gathered in the next lemma.

Lemma 3.2 Assume that $u_{0}, p$, and $q$ fulfil (1.2) and (1.3). Let $u$ be the viscosity solution to (1.1) described in Proposition 3.1. Then $t \longmapsto\|u(t)\|_{L^{\infty}(B)}$ is a non-increasing function and

$$
M_{\infty}:=\lim _{t \rightarrow \infty}\|u(t)\|_{L^{\infty}(B)}>0 .
$$

Proof. Any positive constant being obviously a supersolution to (1.1), the time monotonicity of the $L^{\infty}(B)$-norm of $u$ readily follows from the comparison principle. Next, since $u_{0} \neq \equiv 0$ by (1.2), there is $x_{0} \in B, \varrho>0$, and $m>0$ such that

$$
B_{\varrho}\left(x_{0}\right):=\left\{x \in \mathbb{R}^{N}:\left|x-x_{0}\right|<\varrho\right\} \subset B \quad \text { and } \quad u_{0}(x) \geq m \quad \text { for } \quad x \in B_{\varrho}\left(x_{0}\right) .
$$

Introducing $v_{\lambda}(x):=\lambda^{(p-q) /(p-1-q)} w_{0}\left(\left|x-x_{0}\right| / \lambda\right)$ for $x \in B_{\lambda}\left(x_{0}\right)$ and $\lambda \in(0,1)$ (the function $w_{0}$ being defined in Theorem [1.1), a simple computation shows that $v_{\lambda}$ is a solution to $-\Delta_{p} v_{\lambda}-\left|\nabla v_{\lambda}\right|^{q}=0$ in $B_{\lambda}\left(x_{0}\right)$ with $v_{\lambda}(x)=0 \leq u(x, t)$ for $(x, t) \in \partial B_{\lambda}\left(x_{0}\right) \times(0, \infty)$. Furthermore, if $\lambda=\lambda_{m}:=\min \left\{1-\left|x_{0}\right|,\left(m \alpha / c_{0}\right)^{(p-1-q) /(p-q)}\right\}$, we have $v_{\lambda_{m}}(x) \leq m \leq u_{0}(x)$ for $x \in B_{\lambda_{m}}\left(x_{0}\right)$. The comparison principle [11, Theorem 2.1] then warrants that $u(x, t) \geq v_{\lambda_{m}}(x)$ for $(x, t) \in B_{\lambda_{m}}\left(x_{0}\right) \times(0, \infty)$. In particular, $\|u(t)\|_{L^{\infty}(B)} \geq\left\|v_{\lambda_{m}}\right\|_{L^{\infty}\left(B_{\lambda_{m}}\left(x_{0}\right)\right)}$ for all $t \geq 0$, whence $M_{\infty} \geq\left\|v_{\lambda_{m}}\right\|_{L^{\infty}\left(B_{\lambda_{m}}\left(x_{0}\right)\right)}>0$.

\section{Convergence to steady states}

We introduce the half-relaxed limits

$$
u_{*}(x):=\liminf _{(s, \varepsilon) \rightarrow(t, 0)} u\left(x, \varepsilon^{-1} s\right), \quad x \in \bar{B},
$$

and

$$
u^{*}(x):=\limsup _{(s, \varepsilon) \rightarrow(t, 0)} u\left(x, \varepsilon^{-1} s\right), \quad x \in \bar{B},
$$

which are well-defined and do not depend on $t>0$. Moreover, we infer from the stability theorem (see [10, Lemma 6.1]) that

$$
u^{*} \text { is a viscosity subsolution to } F\left(\nabla z, D^{2} z\right)=0 \text { in } B,
$$




$$
u_{*} \text { is a viscosity supersolution to } F\left(\nabla z, D^{2} z\right)=0 \text { in } B \text {. }
$$

Next we state some useful properties of the half-relaxed limits.

Lemma 4.1 The half-relaxed limits $u_{*}$ and $u^{*}$ enjoy the following properties:

$$
\begin{aligned}
& u_{*} \in W^{1, \infty}(B), \quad u^{*} \in W^{1, \infty}(B), \\
& 0 \leq u_{*}(x) \leq u^{*}(x), \quad x \in \bar{B}, \\
& u_{*} \text { and } u^{*} \text { are radially symmetric and non-increasing, } \\
& u_{*}(0)=u^{*}(0)=M_{\infty}:=\lim _{t \rightarrow \infty}\|u(t)\|_{L^{\infty}(B)}>0, \\
& u_{*}(x)=u^{*}(x)=0 \quad \text { for } x \in \partial B .
\end{aligned}
$$

Proof. By (3.1) there is $L:=\max \left\{A_{0}, W(0)\right\}>0$ such that

$$
u\left(x, \varepsilon^{-1} s\right) \leq u\left(y, \varepsilon^{-1} s\right)+L|x-y| \quad \text { for all }\left(x, y, \varepsilon^{-1} s\right) \in \bar{B} \times \bar{B} \times[0, \infty),
$$

from which we deduce that $u_{*}$ and $u^{*}$ are Lipschitz continuous in $B$ by taking the limsup or liminf in $\varepsilon$ and $s$. This proves (4.3), while (4.4) comes directly from the definition of $u_{*}$ and $u^{*}$ and the facts that $u$ is non-negative, radially symmetric for any $t \geq 0$ and vanishes identically on $\partial B \times(0, \infty)$. The proof of (4.7) uses, in addition, the uniform Lipschitz and $C^{0,1 / 2}$-bounds we have for $u$ in space and time respectively.

In order to prove (4.5), we use Proposition 3.1 there is a decreasing function $W$ such that $W(t) \rightarrow 0$ as $t \rightarrow \infty$ and

$$
u(x, t) \leq u(y, t)+W(t)(|x|-|y|) \quad \text { for } \quad(x, y) \in \bar{B} \times \bar{B} \quad \text { such that } \quad|x| \geq|y| .
$$

Using this inequality with $t=\varepsilon^{-1} s$ and taking the limsup or liminf in $\varepsilon$ and $s$ lead to either $u_{*}(x) \leq u_{*}(y)$ or $u^{*}(x) \leq u^{*}(y)$ for any $(x, y) \in \bar{B} \times \bar{B}$ such that $|x| \geq|y|$ because $W(t) \rightarrow 0$ as $t \rightarrow \infty$, hence to 4.5 .

It remains to show (4.6). To this end, we recall that $M_{\infty}$ is well-defined and positive by (3.13) and first claim that

$$
\lim _{t \rightarrow \infty} u(0, t)=M_{\infty}
$$

Indeed, (4.9) implies

$$
u(x, t) \leq u(0, t)+W(t)|x| \leq u(0, t)+W(t) \leq\|u(t)\|_{L^{\infty}(B)}+W(t), \quad x \in B
$$

whence

$$
\|u(t)\|_{L^{\infty}(B)} \leq u(0, t)+W(t) \leq\|u(t)\|_{L^{\infty}(B)}+W(t),
$$

and (4.10) due to $W(t) \rightarrow 0$ as $t \rightarrow \infty$.

Moreover, by the definition of the half-relaxed limits, we have $u_{*}(0)=u^{*}(0)=M_{\infty}$ and

$$
\left\|u_{*}\right\|_{L^{\infty}(B)} \leq\left\|u^{*}\right\|_{L^{\infty}(B)} \leq M_{\infty} .
$$

This completes the proof of (4.6). 
Now, owing to the monotonicity and radial symmetry of $u_{*}$ and $u^{*}$, there are $r_{*} \in[0,1]$ and $r^{*} \in[0,1]$ such that

$$
\begin{aligned}
& u_{*}(x)=M_{\infty} \text { if }|x| \leq r_{*} \text { and } u_{*}(x)<M_{\infty} \text { if }|x| \in\left(r_{*}, 1\right], \\
& u^{*}(x)=M_{\infty} \text { if }|x| \leq r^{*} \text { and } u^{*}(x)<M_{\infty} \text { if }|x| \in\left(r^{*}, 1\right],
\end{aligned}
$$

Due to (4.4), (4.6), and (4.7), we have

$$
0 \leq r_{*} \leq r^{*}<1
$$

Next, we show that $\Lambda u_{*}$ is a strict supersolution to the stationary equation in a subset of $B$ for $\Lambda>1$.

Lemma 4.2 Fix $\Lambda>1$ and $\delta \in\left(0,1-r_{*}\right)$. Then there are $r_{\delta} \in\left(r_{*}, r_{*}+\delta\right)$ and $\varepsilon_{\delta, \Lambda}>0$ such that $\Lambda u_{*}$ is a viscosity supersolution to $f\left(r, \partial_{r} z, \partial_{r}^{2} z\right)=\varepsilon_{\delta, \Lambda}$ in $\left(r_{\delta}, 1\right)$. In addition, $\varepsilon_{\delta, \Lambda} \rightarrow 0$ as $\Lambda \searrow 1$.

Proof. Fix $\delta \in\left(0,1-r_{*}\right)$. Then, due to (4.3), (4.5), and (4.11), there is $r_{\delta} \in\left(r_{*}, r_{*}+\delta\right.$ ) such that $u_{*}$ is differentiable at $r_{\delta}$ and $\partial_{r} u_{*}\left(r_{\delta}\right)<0$. Since $u_{*}$ is a viscosity supersolution to $f\left(r, \partial_{r} z, \partial_{r}^{2} z\right)=0$ in $(0,1)$, it is also a viscosity supersolution to $f_{0}\left(r, \partial_{r} z, \partial_{r}^{2} z\right)=0$ in $(0,1)$ and it follows from Lemma 2.1 that

$$
\partial_{r} u_{*}(r) \leq r^{(N-1) /(p-1)} \partial_{r} u_{*}(r) \leq r_{\delta}^{(N-1) /(p-1)} \partial_{r} u_{*}\left(r_{\delta}\right)=:-m_{\delta}<0
$$

for a.e. $r \in\left(r_{\delta}, 1\right)$. Integrating and using the continuity of $u_{*}$ we conclude that

$$
u_{*}(r) \leq u_{*}\left(r_{1}\right)-m_{\delta}\left(r-r_{1}\right)
$$

for all $r_{1} \in\left[r_{\delta}, 1\right]$ and $r \in\left[r_{1}, 1\right]$.

Consider $\Lambda>1, \Phi \in \mathcal{C}^{2}\left(\left(r_{\delta}, 1\right)\right)$ and assume that $\Lambda u_{*}-\Phi$ has a local minimum at some $r_{0} \in\left(r_{\delta}, 1\right)$. Then $u_{*}-(\Phi / \Lambda)$ has a local minimum at $r_{0}$ and (4.2) implies

$$
\begin{aligned}
& -\frac{1}{r_{0}^{N-1}} \partial_{r}\left(r^{N-1}\left|\partial_{r}\left(\frac{\Phi}{\Lambda}\right)\right|^{p-2} \partial_{r}\left(\frac{\Phi}{\Lambda}\right)\right)\left(r_{0}\right)-\left|\partial_{r}\left(\frac{\Phi}{\Lambda}\right)\left(r_{0}\right)\right|^{q} \geq 0, \\
& -\frac{1}{r_{0}^{N-1}} \partial_{r}\left(r^{N-1}\left|\partial_{r} \Phi\right|^{p-2} \partial_{r} \Phi\right)\left(r_{0}\right)-\Lambda^{p-1-q}\left|\partial_{r} \Phi\left(r_{0}\right)\right|^{q} \geq 0 .
\end{aligned}
$$

Thus, we have

$$
-\frac{1}{r_{0}^{N-1}} \partial_{r}\left(r^{N-1}\left|\partial_{r} \Phi\right|^{p-2} \partial_{r} \Phi\right)\left(r_{0}\right)-\left|\partial_{r} \Phi\left(r_{0}\right)\right|^{q} \geq\left(\Lambda^{p-1-q}-1\right)\left|\partial_{r} \Phi\left(r_{0}\right)\right|^{q} .
$$

Now, since $\Lambda u_{*}-\Phi$ has a local minimum at $r_{0}$, we infer from (4.14) that, for $r \in\left[r_{0}, r_{0}+\eta\right]$ with $\eta>0$ small enough,

$$
u_{*}\left(r_{0}\right) \leq \frac{\Phi\left(r_{0}\right)}{\Lambda}+u_{*}(r)-\frac{\Phi(r)}{\Lambda} \leq \frac{\Phi\left(r_{0}\right)}{\Lambda}+u_{*}\left(r_{0}\right)-m_{\delta}\left(r-r_{0}\right)-\frac{\Phi(r)}{\Lambda} .
$$

Hence,

$$
\frac{\Phi(r)}{\Lambda}-\frac{\Phi\left(r_{0}\right)}{\Lambda} \leq-m_{\delta}\left(r-r_{0}\right)
$$


and thus

$$
\frac{1}{\Lambda} \partial_{r} \Phi\left(r_{0}\right) \leq-m_{\delta}<0
$$

which implies $\left|\partial_{r} \Phi\left(r_{0}\right)\right| \geq \Lambda m_{\delta}$. Consequently, (4.15) becomes

$$
f\left(r_{0}, \partial_{r} \Phi\left(r_{0}\right), \partial_{r}^{2} \Phi\left(r_{0}\right)\right) \geq\left(\Lambda^{p-1-q}-1\right) \Lambda^{q} m_{\delta}^{q}=: \varepsilon_{\delta, \Lambda}>0,
$$

which ends the proof.

We are now able to prove that the half-relaxed limits $u_{*}$ and $u^{*}$ coincide.

Lemma 4.3 We have $u_{*}=u^{*}$ on $\bar{B}$.

Proof. We fix $\Lambda>1>\lambda>0$ such that $\lambda>r_{*}$ and

$$
\delta:=\frac{M_{\infty}}{\left\|\nabla u_{*}\right\|_{L^{\infty}(B)}}\left(1-\lambda^{(p-q) /(p-1-q)}\right) \in\left(0, \lambda-r_{*}\right) .
$$

Defining now

$$
U(r):=\Lambda u_{*}(r), \quad r \in[0,1], \quad \text { and } \quad V(r):=\lambda^{(p-q) /(p-1-q)} u^{*}\left(\frac{r}{\lambda}\right), \quad r \in[0, \lambda],
$$

we obtain due to 4.13)

$$
U(r) \geq u_{*}(r)=M_{\infty} \geq V(r) \quad \text { for } r \in\left[0, r_{*}\right] .
$$

Furthermore, we infer from the Lipschitz continuity of $u_{*}$ that, for $r \in\left(r_{*}, r_{*}+\delta\right]$,

$$
\begin{aligned}
U(r) & \geq u_{*}(r) \geq u_{*}\left(r_{*}\right)-\left\|\nabla u_{*}\right\|_{L^{\infty}(B)}\left|r-r_{*}\right| \\
& =M_{\infty}-\left\|\nabla u_{*}\right\|_{L^{\infty}(B)}\left|r-r_{*}\right| \geq M_{\infty}-\delta\left\|\nabla u_{*}\right\|_{L^{\infty}(B)} \\
& \geq \lambda^{(p-q) /(p-1-q)} M_{\infty} \geq V(r) .
\end{aligned}
$$

Recalling (4.16), we have thus shown that

$$
U(r) \geq V(r) \quad \text { for } \quad r \in\left[0, r_{*}+\delta\right] .
$$

Next, we define $I_{\lambda}:=\left(r_{*}+\delta, \lambda\right)$. On the one hand, $V$ is a viscosity subsolution to $f\left(r, \partial_{r} z, \partial_{r}^{2} z\right)=0$ in $I_{\lambda}$. Indeed, take $\Phi \in \mathcal{C}^{2}\left(I_{\lambda}\right)$ and assume that $V-\Phi$ has a local maximum at $r_{1} \in I_{\lambda}$. Then $u^{*}-\Psi$ has a local maximum at $r_{1} / \lambda$, where $\Psi(r):=\lambda^{-(p-q) /(p-1-q)} \Phi(\lambda r)$ for $r \in\left(\left(r_{*}+\delta\right) / \lambda, 1\right)$. Owing to (4.1), we obtain

$$
f\left(\frac{r_{1}}{\lambda}, \partial_{r} \Psi\left(\frac{r_{1}}{\lambda}\right), \partial_{r}^{2} \Psi\left(\frac{r_{1}}{\lambda}\right)\right) \leq 0 .
$$

Consequently,

$$
\begin{aligned}
0 & \geq \lambda^{q /(p-1-q)} f\left(\frac{r_{1}}{\lambda}, \lambda^{-1 /(p-1-q)} \partial_{r} \Phi\left(r_{1}\right), \lambda^{1-1 /(p-1-q)} \partial_{r}^{2} \Phi\left(r_{1}\right)\right) \\
& =-(p-1)\left|\partial_{r} \Phi\left(r_{1}\right)\right|^{p-2} \partial_{r}^{2} \Phi\left(r_{1}\right)-\frac{N-1}{r_{1}}\left|\partial_{r} \Phi\left(r_{1}\right)\right|^{p-2} \partial_{r} \Phi\left(r_{1}\right)-\left|\partial_{r} \Phi\left(r_{1}\right)\right|^{q} \\
& =f\left(r_{1}, \partial_{r} \Phi\left(r_{1}\right), \partial_{r}^{2} \Phi\left(r_{1}\right)\right)
\end{aligned}
$$


and $V$ is a viscosity subsolution to $f\left(r, \partial_{r} z, \partial_{r}^{2} z\right)=0$ in $I_{\lambda}$. On the other hand, it follows from Lemma 4.2 that $U$ is a viscosity supersolution to $f\left(r, \partial_{r} z, \partial_{r}^{2} z\right)=\varepsilon_{\delta, \Lambda}$ in $I_{\lambda}$ with some $\varepsilon_{\delta, \Lambda}>0$. As furthermore $V(r)=0 \leq U(r)$ for $r=\lambda$ and $U(r) \geq V(r)$ for $r=r_{*}+\delta$ due to (4.17), we conclude that

$$
U(r) \geq V(r) \quad \text { for } r \in\left[r_{*}+\delta, \lambda\right]
$$

by [10, Section $5 \mathrm{C}]$. Using (4.17), we end up with

$$
\Lambda u_{*}(r) \geq \lambda^{(p-q) /(p-1-q)} u^{*}\left(\frac{r}{\lambda}\right) \quad \text { for } r \in[0, \lambda] .
$$

Letting now $\Lambda \searrow 1$ and $\lambda \nearrow 1$, we conclude $u_{*} \geq u^{*}$ in $[0,1]$ which, together with (4.4), implies $u^{*}=u_{*}$.

Finally, we prove Theorem 1.3 .

Proof of Theorem 1.3 .

Defining $u_{\infty}:=u_{*}=u^{*}$ by Lemma 4.3, (4.1), (4.2), and Lemma 4.1 imply that $u_{\infty}$ is a radially symmetric, non-increasing, and Lipschitz continuous viscosity solution to $F\left(\nabla z, D^{2} z\right)=0$ in $B$ satisfying $u_{\infty}=0$ on $\partial B$. Moreover, $\left\|u_{\infty}\right\|_{L^{\infty}(B)}=M_{\infty}>0$ due to (4.6). Hence, owing to Theorem [1.1, there is a unique $\vartheta \in[0,1)$ such that $u_{\infty}=w_{\vartheta}$.

In particular, the equality $u_{*}=u^{*}$ and the definition of $u_{*}$ and $u^{*}$ provide the uniform convergence of $u(t)$ towards $u^{*}=w_{\vartheta}$ in every compact subset of $B$ as $t \rightarrow \infty$, see [3, Lemme 4.1] or [2, Lemma V.1.9]. Combining this local convergence with (4.3) and (4.7) gives

$$
\lim _{t \rightarrow \infty}\left\|u(t)-w_{\vartheta}\right\|_{\mathcal{C}(\bar{B})}=0
$$

and the claim is proved.

\section{Acknowledgements}

The authors would like to thank Olivier Ley for helpful discussions and comments. Part of this work was done during visits of Ph. Laurençot to the Fachbereich Mathematik of the Universität Duisburg-Essen and of C. Stinner to the Institut de Mathématiques de Toulouse, Université Paul Sabatier - Toulouse III. We would like to express our gratitude for the invitation, support, and hospitality.

\section{References}

[1] J.M. Arrieta, A. Rodriguez-Bernal and Ph. Souplet, Boundedness of global solutions for nonlinear parabolic equations involving gradient blow-up phenomena, Ann. Sc. Norm. Super. Pisa Cl. Sci. (5) 3 (2004), 1-15.

[2] M. Bardi and I. Capuzzo-Dolcetta, Optimal Control and Viscosity Solutions of HamiltonJacobi-Bellman Equations, Systems Control Found. Appl., Birkhäuser, Boston, 1997. 
[3] G. Barles, Solutions de Viscosité des Equations d'Hamilton-Jacobi, Mathématiques \& Applications 17, Springer-Verlag, Berlin, 1994.

[4] G. Barles and J. Busca, Existence and comparison results for fully nonlinear degenerate elliptic equations without zeroth-order term, Comm. Partial Differential Equations 26 (2001), 2323-2337.

[5] G. Barles and F. Da Lio, On the generalized Dirichlet problem for viscous HamiltonJacobi equations, J. Math. Pures Appl. 83 (2004), 53-75.

[6] G. Barles, G. Díaz, and J.I. Díaz, Uniqueness and continuum of foliated solutions for a quasilinear elliptic equation with a non lipschitz nonlinearity, Comm. Partial Differential Equations 17 (1992), 1037-1050.

[7] G. Barles and B. Perthame, Exit time problems in optimal control and vanishing viscosity method, SIAM J. Control Optim. 26 (1988), 1133-1148.

[8] G. Barles and P.E. Souganidis, On the large time behaviour of solutions of HamiltonJacobi equations, SIAM J. Math. Anal. 31 (2000), 925-939.

[9] S. Benachour, S. Dăbuleanu-Hapca, and Ph. Laurençot, Decay estimates for a viscous Hamilton-Jacobi equation with homogeneous Dirichlet boundary conditions, Asymptot. Anal. 51 (2007), 209-229.

[10] M.G. Crandall, H. Ishii, and P.-L. Lions, User's guide to viscosity solutions of second order partial differential equations, Bull. Amer. Math. Soc. (N.S.) 27 (1992), 1-67.

[11] Y. Giga, S. Goto, H. Ishii, and M.-H. Sato, Comparison principle and convexity preserving properties for singular degenerate parabolic equations on unbounded domains, Indiana Univ. Math. J. 40 (1991), 443-470.

[12] B.H. Gilding, M. Guedda and R. Kersner, The Cauchy problem for $u_{t}=\Delta u+|\nabla u|^{q}$, J. Math. Anal. Appl. 284 (2003), 733-755.

[13] O.A. Ladyženskaja, V.A. Solonnikov, and N.N Ural'ceva, Linear and Quasi-linear Equations of Parabolic Type, Amer. Math. Soc., Providence, 1968.

[14] Ph. Laurençot, Convergence to steady states for a one-dimensional viscous HamiltonJacobi equation with Dirichlet boundary conditions, Pacific J. Math. 230 (2007), 347364 .

[15] G. Namah and J.-M. Roquejoffre, Remarks on the long time behaviour of the solutions of Hamilton-Jacobi equations, Comm. Partial Differential Equations 24 (1999), 883-893.

[16] J.-M. Roquejoffre, Convergence to steady states or periodic solutions in a class of Hamilton-Jacobi equations, J. Math. Pures Appl. (9) 80 (2001), 85-104.

[17] Ph. Souplet, Gradient blow-up for multidimensional nonlinear parabolic equations with general boundary conditions, Differential Integral Equations 15 (2002), 237-256. 
[18] Ph. Souplet and Q.S. Zhang, Global solutions of inhomogeneous Hamilton-Jacobi equations, J. Analyse Math. 99 (2006), 355-396.

[19] C. Stinner, Convergence to steady states in a viscous Hamilton-Jacobi equation with degenerate diffusion, preprint (2009).

[20] T. Tabet Tchamba, Large time behavior of solutions of viscous Hamilton-Jacobi equations with superquadratic Hamiltonian, preprint (2009).

[21] T.I. Zelenyak, Stabilization of solutions of boundary value problems for a second order parabolic equation with one space variable, Differential Equations 4 (1968), 17-22. 